P0159

DYNAMIC GLARE EVALUATION ALONG A ROUTE

Vincent Boucher et al.

DOI 10.25039/x46.2019.PO159

from

CIE x046:2019

Proceedings

of the

29th CIE SESSION

Washington D.C., USA, June 14 - 22, 2019

(DOI 10.25039/x46.2019)

The paper has been presented at the 29th CIE Session, Washington D.C., USA, June 14-22, 2019. It has not been peer-reviewed by CIE.

(C) CIE 2019

All rights reserved. Unless otherwise specified, no part of this publication may be reproduced or utilized in any form or by any means, electronic or mechanical, including photocopying and microfilm, without permission in writing from CIE Central Bureau at the address below. Any mention of organizations or products does not imply endorsement by the CIE.

This paper is made available open access for individual use. However, in all other cases all rights are reserved unless explicit permission is sought from and given by the CIE.

CIE Central Bureau

Babenbergerstrasse 9

A-1010 Vienna

Austria

Tel.: +4317143187

e-mail: ciecb@cie.co.at

www.cie.co.at 


\title{
DYNAMIC GLARE EVALUATION ALONG A ROUTE
}

\author{
Vincent Boucher ${ }^{1}$, Florian Greffier ${ }^{1}$, Fabrice Fournela ${ }^{1}$, Romain Dronneau ${ }^{1}$. \\ ${ }^{1}$ Cerema, Direction Ouest, 23 avenue Amiral Chauvin, F-49130, Les Ponts-de-Cé, FRANCE. \\ vincent.boucher@cerema.fr
}

DOI 10.25039/x46.2019.PO159

\begin{abstract}
Road lighting measurements are performed capturing low luminances and high luminances in the same image. It is known as High Dynamic Range (HDR) imagery and the technique used here is based on four synchronous CMOS cameras, each dedicated to one luminance range. After geometric and photometric calibration, an image processing algorithm constructs the final 20-bit HDR image. Then threshold increment and visual adaption models are computed to evaluate glare dynamically along a route. For each model, we discuss about the different parameters and their validity in moving situation.
\end{abstract}

Keywords: High Dynamic Range, Threshold Increment, Visual adaption, Road Lighting

\section{Purpose}

Evaluating road lighting performances is possible nowadays by mean of digital cameras known as Imaging Luminance Measuring Devices (ILMD). Normative standards can be easily checked and glare evaluation can be computed as soon as sources luminance are measured. However with classical cameras, the major part of sources is saturated due to their poor dynamic range. Accessing to glare in an image implies no saturation in light sources. Several techniques exist for extending image sensor dynamic range. A simple one, is to capture several images with different exposure times. It works fine as far as nothing change in the scene i.e. in static. In a moving frame, images have to captured in the same time. So we developed an imaging system based on four synchronous cameras, each dedicated to one luminance range. But it leads to a reconstruction issue : images are no more superimposable since cameras each have their own point of view (see figure 1). To registrate four synchronous images, we performed a stereo-like calibration in two dimensions. This step permits to facilitate the pixels combination and to construct HDR images containing the whole scene illumination range.

The human eye has a remarkable dynamic range that enables it to interpret scenes under many illumination conditions. However vision can be deteriorated especially at night in presence of light sources in the field of view. This effect is known as disability glare and can be modelled from a veiling luminance calculation. A first glare model will be applied on HDR images to evaluate glare along a boulevard. On the other hand, the eye dynamic range is also due to its ability to adjust its sensitivity with incoming light. It is known as visual adaption and is modelled through cones and rods response curves. A second glare model based on these considerations will be applied on the same image and compared with the previous results.

\section{Calibrated HDR Imaging System}

Several techniques and architectures have been proposed for extending image sensor dynamic range(Decker, 1998, Kavadias, 2000, Kleinfelder, 2001, Knight, 1983, Nayar, 2000, Sayag, 1991). One way is to capture several images with different exposure time: short integration time capture high light regions and long integration time capture low light regions. Combination of all images into HDR image is then required via an HDR reconstruction algorithm (Reinhard, 2002, 2010). The Signal over Noise Ratio (SNR) is not affected and maintained over extended dynamic range. The response stays linear and the same for each 
pixel, so conventional photometric and colorimetric calibrations can be performed. One issue is synchronization of images : our solution is a multiple capture technique using four CMOS cameras (Figure 1), each dedicated to one luminance range.

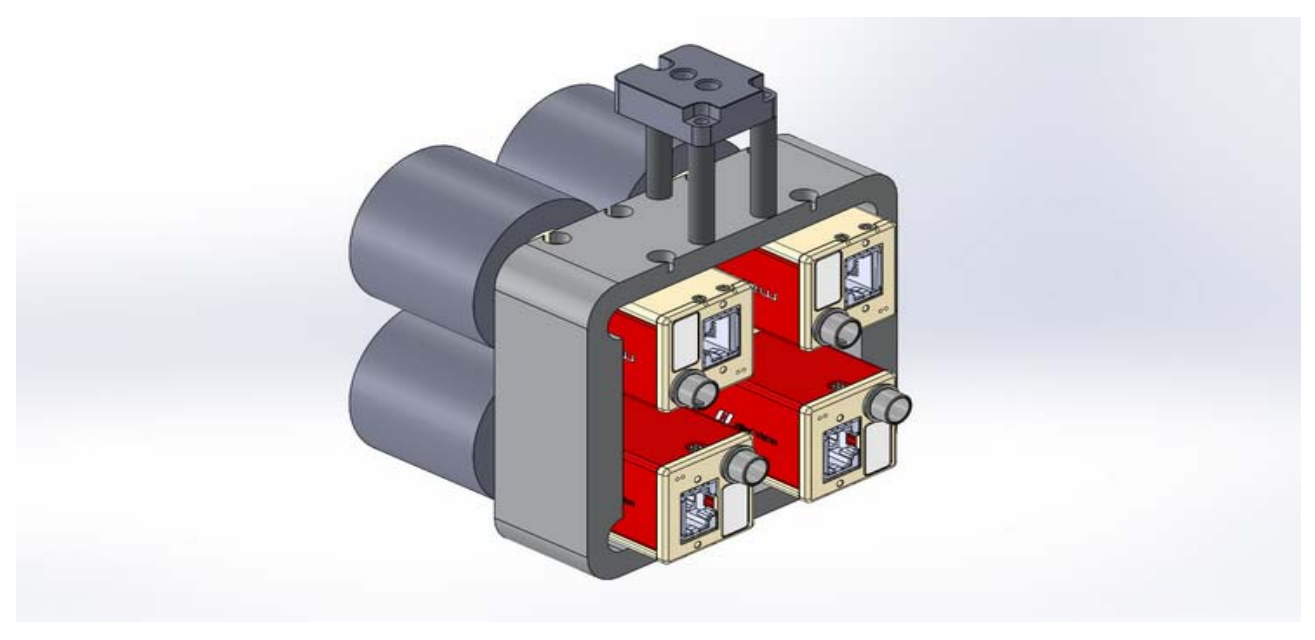

Figure 1 - Four cameras assembly

The synchronization issue is solved by using hardware triggered cameras. The time lag has been measured inferior to $1 \mathrm{~ms}$. The major issue of this configuration is their different points of view and classical image registration algorithms cannot be applied. The setup can be assimilated to a multiple stereoscopic system and a rigorous geometric calibration is needed to allow the HDR reconstruction. We used stereo imaging tools and derived a specific process to characterize geometric parameters:

A camera can be completely modelled by a list of internal parameters known as intrinsic parameters and the relative position of a set of cameras can be described with extrinsic parameters. Using the normalized (pinhole) image projection, a point in the camera reference frame can be projected on the image plane according to the intrinsic parameters:

- Focal length: The horizontal and vertical focal lengths in pixels $f_{u}, f_{v}$.

- Principal point: The principal point coordinates in the image $\boldsymbol{u}_{\mathbf{0}}, \boldsymbol{v}_{\boldsymbol{o}}$.

The pixel coordinates are related to the world coordinates through a linear equation defined by a matrix $\mathrm{M}$ known as the camera matrix:

$$
M=\left[\begin{array}{ccc}
f_{u} & 0 & u_{0} \\
0 & f_{v} & v_{0} \\
0 & 0 & 1
\end{array}\right]
$$

Using optical lenses of $12.5 \mathrm{~mm}$ with Mako G234C cameras (10-bit CMOS, resolution : $1936 \mathrm{x}$ 1216, pixel size : $5.86 \mu \mathrm{m})$, theoretical focal length is 2133 pixels and principal point coordinates are 968 and 608, compatible with the calculated results presented in Table 1. These results will be used to compute extrinsic parameters. 
Table 1 - Intrinsic parameters of cameras

\begin{tabular}{|c|c|c|c|c|}
\hline & CAM1 & CAM2 & CAM3 & CAM4 \\
\hline $\begin{array}{c}\text { Focal length } \\
{[\mathrm{fu} ; \mathrm{fv}] \text { in pixels }}\end{array}$ & {$[2161.5 ; 2162.5]$} & {$[2155.4 ; 2156.1]$} & {$[2164.6 ; 2165.2]$} & {$[2156.6 ; 2157.4]$} \\
\hline $\begin{array}{c}\text { Principal point } \\
{[\mathrm{u} 0 ; \mathrm{v} 0] \text { in pixels }}\end{array}$ & {$[961.7 ; 627.4]$} & {$[959.6 ; 637.3]$} & {$[954.1 ; 640.2]$} & {$[979.6 ; 621.2]$} \\
\hline
\end{tabular}

Extrinsic parameters are defined by a rotation matrix $R$ and a translation vector $T$. Consider two cameras as in stereoscopic calibration and a point of the calibration grid. Its two coordinates vectors $X_{1}$ and $X_{2}$ in the first and second camera reference frames respectively are related to each other through the rigid motion transformation:

$$
X_{2}=R^{*} X_{1}+T
$$

where $R$ can be expressed as a rotation vector $r$ presented in Table 2. The optical lenses have a diameter of $45 \mathrm{~mm}$ and are placed side by side. A translation of this order of magnitude is expected and found relatively close (see the first $T$ coordinate for horizontal dimension and the second coordinate for vertical dimension).

\section{Table 2 - Extrinsic parameters of cameras}

\begin{tabular}{|l|c|c|c|c|}
\hline & CAM1/CAM2 & CAM3/CAM4 & CAM1/CAM3 & CAM2/CAM4 \\
\hline$\left[\mathrm{r}_{\mathrm{x} ;} ; \mathrm{r}_{\mathrm{r}} ; \mathrm{r}_{z}\right](\mathrm{mrad})$ & {$[2.37 ; 4.32 ; 0.76]$} & {$[0.31 ;-1.63 ; 1.56]$} & {$[-4.40 ; 11.2 ;-0.57]$} & {$[6.87 ; 4.73 ; 0.22]$} \\
\hline$\left[\mathrm{T}_{\mathrm{x} ;} ; \mathrm{T}_{\mathrm{y} ;} ; \mathrm{T}_{\mathrm{z}}\right](\mathrm{mm})$ & {$[-46.2 ; 0.15 ; 0.88]$} & {$[-46.3 ;-0.54 ;-1.19]$} & {$[0.50 ;-45.4 ; 3.30]$} & {$[0.38 ;-46.1 ; 0.95]$} \\
\hline
\end{tabular}

This process has been applied at first in the horizontal dimension (see the first two columns in Table 2) to obtain stereo rectified images (with epipolar lines matching with the horizontal scanned lines). Then a second process in the vertical dimension (the last two columns in Table 2) results in doubly-rectified images. This specific registration permits to retrieve a point source from one camera in the three others within a circle of twenty pixels radius corresponding to a $0.5^{\circ}$ field of view. This is a key point to construct HDR images.

Concerning the photometric calibration, the first step is to set the exposure times and gains in the four cameras as well as objective $\mathrm{F}$-number to obtain a total luminance range between less than $1 \mathrm{~cd} / \mathrm{m}^{2}$ and $10^{5} \mathrm{~cd} / \mathrm{m}^{2}$. To avoid motion blur, integration time has to be set up inferior or equal to $10 \mathrm{~ms}$. This requirement is obvious for high illumination cameras but implies the use of gain to have access to low luminances. The most sensitive camera has the minimal F-Number 0.95, an exposure time of $10 \mathrm{~ms}$ with a gain of $20 \mathrm{~dB}$. These parameters can be expressed in terms of Exposure Value (EV). Successive cameras are then set by adding $5 \mathrm{EV}$ with respect to the settings of the previous camera. A theoretical global dynamic range of $20 \mathrm{EV}$ is obtained. Precautions are also taken to correct non-uniformity due to optics and sensors, recording a flat-field and a dark image. The useful signal is then computed from raw signal with the expression:

$$
\text { signal }=\frac{\text { signal }_{\text {raw }}-\text { offset }}{\text { flat }- \text { offset }}
$$




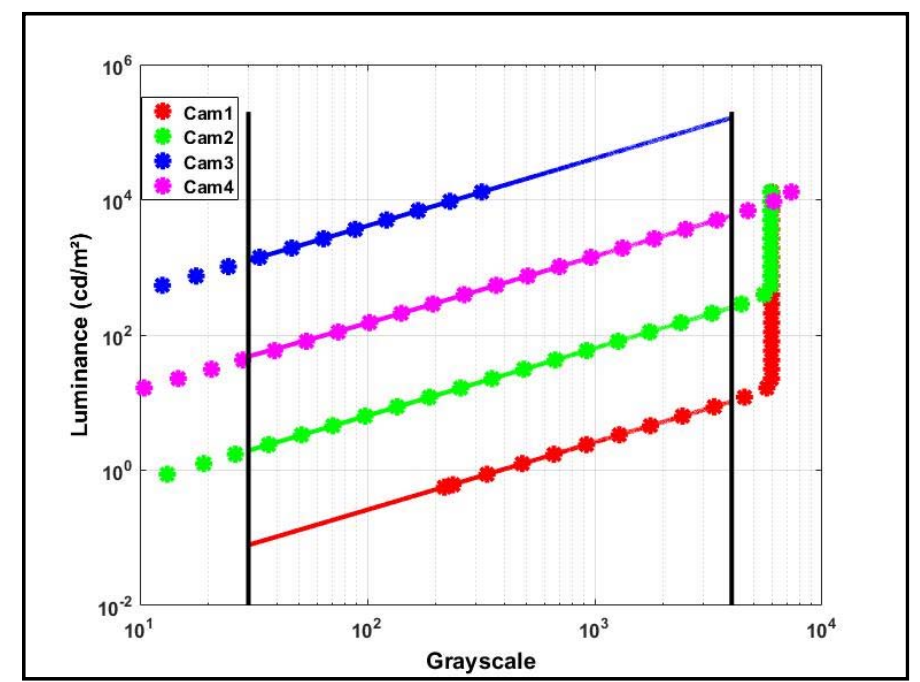

Figure 2 - Photometric calibration

Once all cameras parameters have been fixed, each camera is then calibrated using an integrating sphere delivering luminances from 0 to $13000 \mathrm{~cd} / \mathrm{m}^{2}$. Figure 2 presents photometric calibrations from signals computed according to expression (3). Linear functions are defined between a minimal grayscale value pix $x_{\text {in }}=30$ (twice the offset) and a maximal value pix $\max =4000$ (represented by the two black lines). These limits will be used to define under-exposed and over-exposed pixels in the HDR construction algorithm. The photometric calibration finally leads to a full range from $0.1 \mathrm{~cd} / \mathrm{m}^{2}$ to $100000 \mathrm{~cd} / \mathrm{m}^{2}$.
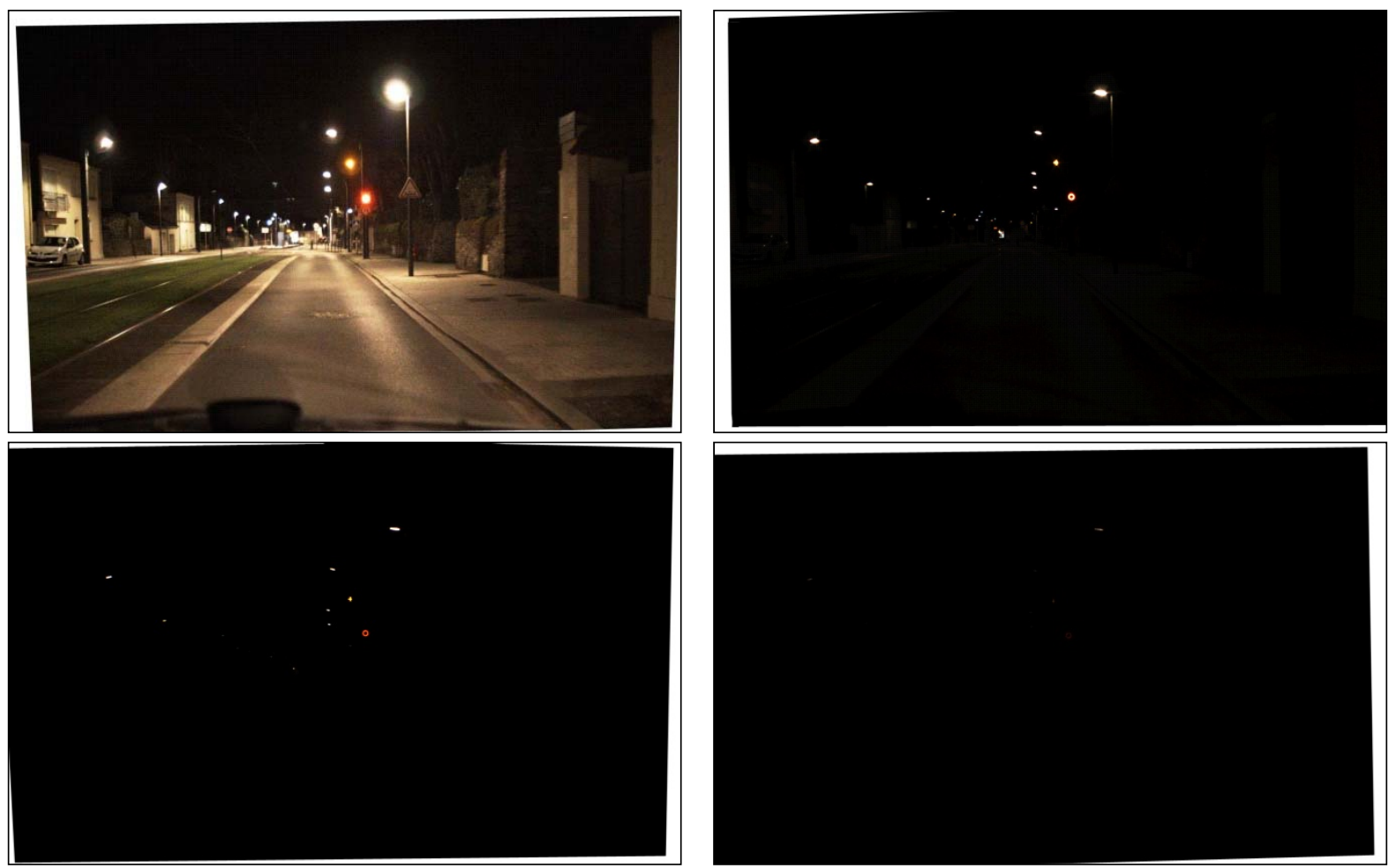

Figure 3 - Quadruplet acquired with the 4 camera system.

Considering a quadruplet of images (IMG $\mathrm{IMM}_{1}$ to $\mathrm{IMG}_{\mathrm{CAM} 4}$ ), the first step is to apply the double rectification (see blank edges in Figure 3 ) provided by the geometric calibration to facilitate the pixel matching. The image with the maximum of well exposed pixels is defined as the main image. From this latter, a 'Hole Filling' algorithm replaces saturated zones where the 
measurement has failed by zones acquired in the other images where the measurement is valid (details in Boucher,2017). The complete process lead to a 20-bit dynamic range image without motion blur. Figure 4 presents the final HDR image of course tone mapped to be presented hereafter.

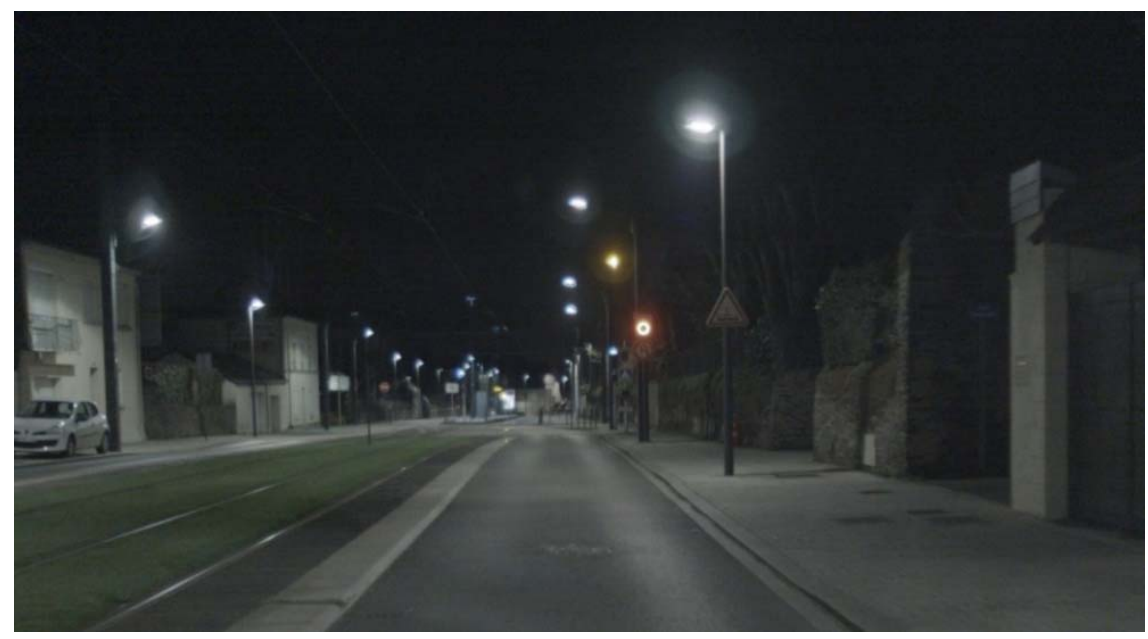

Figure 4 - Final 20-bit HDR image

\section{Glare models}

Threshold Increment $(\mathrm{TI})$ is a well known model to evaluate disability glare under road lighting conditions. But it was defined as a night-time static model with quasi-punctual sources. We can wonder about the values of its parameters and its relevancy in a moving framework. We also propose an alternative model based on vision mechanism. Its philosophy is to adjust sensor response to the incoming light like our eyes do in real life. We finally compare the two models along a boulevard and discuss about parameters value and validity in dynamic.

\subsection{Threshold Increment}

We have computed CIE glare calculations (CIE, 1976) on HDR images. Glare which impairs the vision of objects is termed disability glare. It is a degradation of visual performance caused by a reduction of contrast. It can occur directly, by reducing the contrast between an object and its background or indirectly by affecting the eye caused by light scattering within the eye. This overlying scattered light is usually described as a veiling luminance $\left(L_{\text {veil }}\right)$. The magnitude of the veiling luminance depends on the intensity and distance of the glare source which together determine the relevant parameter $E_{\text {glare }}$, the illuminance at the eye caused by the glare source, and the angle between the glare source and the line of sight $\theta$. The method for the evaluation of disability glare is based on the Holladay formula (CIE, 1976), and the equivalent veiling luminance $L_{\text {veil }}$ in $\mathrm{cd} / \mathrm{m}^{2}$ may be expressed as

$$
L_{\text {veil }}=K \frac{E_{\text {glare }}}{\theta^{2}}
$$

where

$K \quad$ is taken as 10 when $\theta$ is expressed in degrees. The value of $K$ is influenced by a number of factors of which the age of the observer is the most significant;

Eglare the illumination on the observer's eye produced by the glare source in the plane perpendicular to the line of sight in lux;

$\theta \quad$ the angle between the centre of the glare source and the line of sight. 
The exponent of $\theta$ is valid from about $1^{\circ}$ to $30^{\circ}$. More refined expressions exist to extend the angle domain between $0.1^{\circ}$ and $100^{\circ}$ (CIE, 2002) but it is not the purpose here and we keep the equation (4) for simplicity. To apply this relation to our images, we have to calculate $L_{\text {veil }}$ in equation (4) as an image (Porsch, 2014). From a point source pixel, the angle $\theta$ can be defined for all pixels in the image. $\theta$ is related to the imaging resolution and the lens type. In our case the measuring cone of one squared pixel is $0.023 \%$ pix. Moreover $L_{\text {veil }}$ also depends on $E_{\text {glare }}$ the vertical illuminance on a plane normal to the line of sight of observer's eye. It can be retrieved for any sources from the measured luminance of the point source by the expression :

$$
E_{\text {glare }}=L_{S} \cdot \Omega_{S} \cdot \cos \theta_{S}
$$

where

$$
\begin{aligned}
& L s \quad \text { the pixel-wise luminance of the pixel source }\left(\mathrm{cd} / \mathrm{m}^{2}\right) \\
& \Omega s \quad \text { the solid angle of the pixel source }(\mathrm{sr}) ; \\
& \theta s \quad \text { the pixel source angle (degree). }
\end{aligned}
$$

Combining equation (4) with equation (5), the veiling luminance can be performed by summation over all the pixel sources:

$$
L_{v e i l}=\frac{K}{\theta^{2}} \cdot \sum_{x, y} L_{S_{x, y}} \cdot \Omega_{S_{x, y}} \cdot \cos \theta_{S_{x, y}} \quad \text { for }(x, y) \in \text { source }
$$

where

$x, y \quad$ coordinates of all pixel sources in the image.

In road lighting considerations (i.e. at night), the amount of disability glare can be found by calculating the threshold increment (TI) of an object which is in use to signify the visual task of a driver on the road. The $\mathrm{TI}$ is expressed as the following ratio:

$$
T I=\beta \frac{L_{\text {veil }} \alpha}{L_{\text {adapt }}} \quad \%
$$

where
Lveil the veiling luminance;
Ladapt the adaptation luminance (usually measured on the road surface); here we chose the average luminance in the grid defined by 13201 standard.
$\alpha, \beta$ constants related to $L_{\text {adapt }}$ values (CIE, 1976). Here $\alpha=65$ and $\beta=0.8$

Our data are constituted of thousands of pixels and an issue is to define which ones belong to a glare source. One answer is to say "all pixels are punctual sources and contribute to the veiling luminance". But it is computer time consuming because each pixel has to considered to calculate the veiling luminance. Reducing the image size by 4 leads to a computation time of $366 \mathrm{~s}$ (Intel Core i7 @3.40GHz) for 204 images. Figure 5 presents the TI along a route considering the driver's gaze at the grid centre. Each source passing in the field of view creates a curve rebound. At the windshield boundary, their excentricity is about $20^{\circ}$ and their contribution to $L_{\text {veil }}$ are weak and lead to a TI value under 15 suggesting a comfortable road lighting installation. 


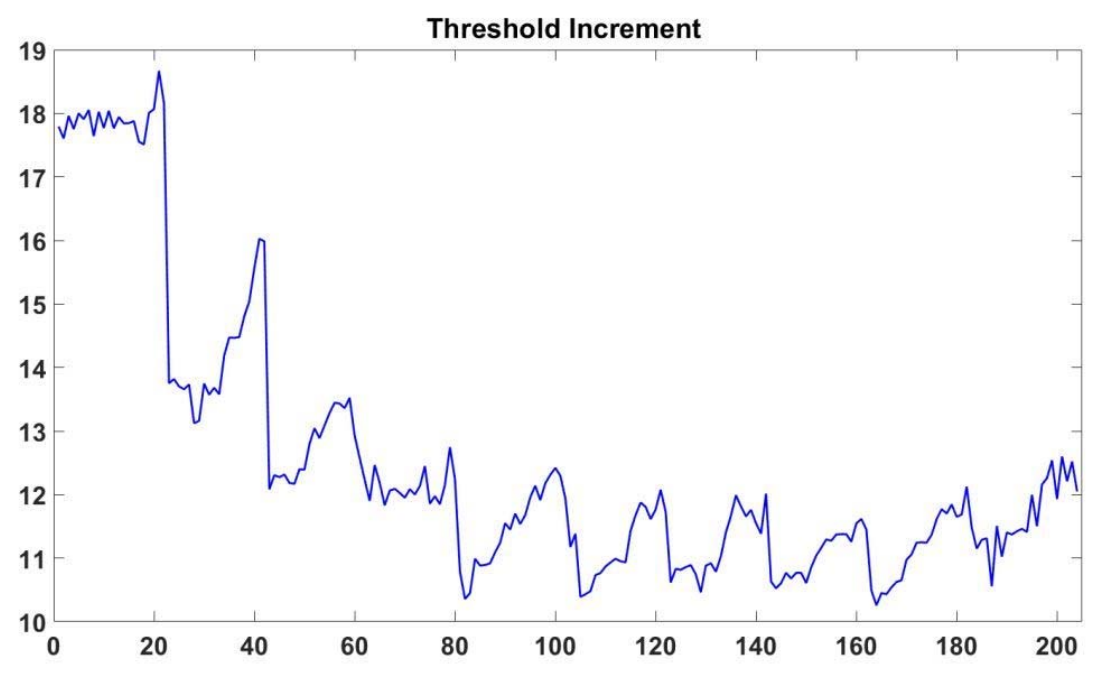

Figure 5 - Threshold Increment along the boulevard

\subsection{Visual Adaption}

Another way to evaluate glare is to identify saturated retinal photoreceptors. The model reproduces visual adaption mechanism : adjust sensor responses to the incoming light. To simulate these adjustments in viewed intensity, we use part of a tone reproduction operator (Pattanaik, 2000). It offers a way of reproducing these time-dependent adaption mechanisms taking place in eye. The visual adaptation model needs the acquisition frequency and the luminance map. For each scene pixel, the model computes retina-like response signals for cone and rod luminance (Boucher, 2015). The static response produces the curve for cones shown in Figure 6. These plots of cone response vs. luminances were drawn with fixed adaptation luminance amounts from $2.10^{-5} \mathrm{~cd} / \mathrm{m}^{2}$ to $2.10^{+7} \mathrm{~cd} / \mathrm{m}^{2}$. Crosses and circles mark the response to adaptation luminance and saturation level respectively. Note that cones adapted above about $10^{+5} \mathrm{~cd} / \mathrm{m}^{2}$ are saturated, with little or no response. The dynamic model will vary only in the horizontal position to mimic the adaptation.

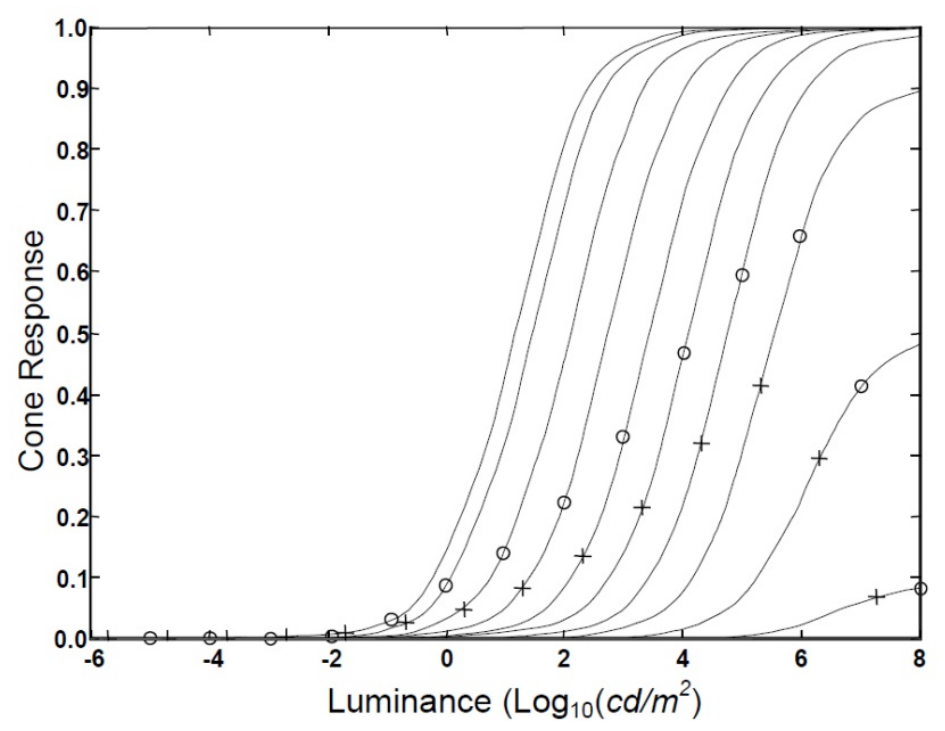

Figure 6 - Model of Cone response (courtesy of Pattanaik,2000)

As output, the visual adaptation model gives the saturated and underexposed zones in relation with cones and rods response of Figure 6 . A pixel (seen as a retinal photoreceptor) is considered to be overexposed if its luminance is superior to five times the adaptation 
luminance. To permit a comparison with TI calculations, Ladapt has been defined as the previous section. Figure 7 presents the two models results along the same boulevard under road lighting. TI curve is normalized with respect to its maximum (in blue). Process time for the same data is reduced at 105 seconds. The visual adaption curve corresponds to the percentage of overexposed photoreceptors over the well-exposed. As can be seen in Figure 7 , visual adaption curve follows the same evolution than TI curve but is more sensitive to sources passage outside the field of view. The previous judgment about comfort is not so definitive regarding the visual adaption curve peaks.

Thus each model raises questions : for the TI model, it has been defined as a static model and what about its validity using it dynamically? For the adaption model, no gaze data are taking into account, nor sources excentricity. Is it relevant with a glare model? This latter leads a relative quantity (overexposed photoreceptor percentage) and need to be scaled with respect to glare perception.

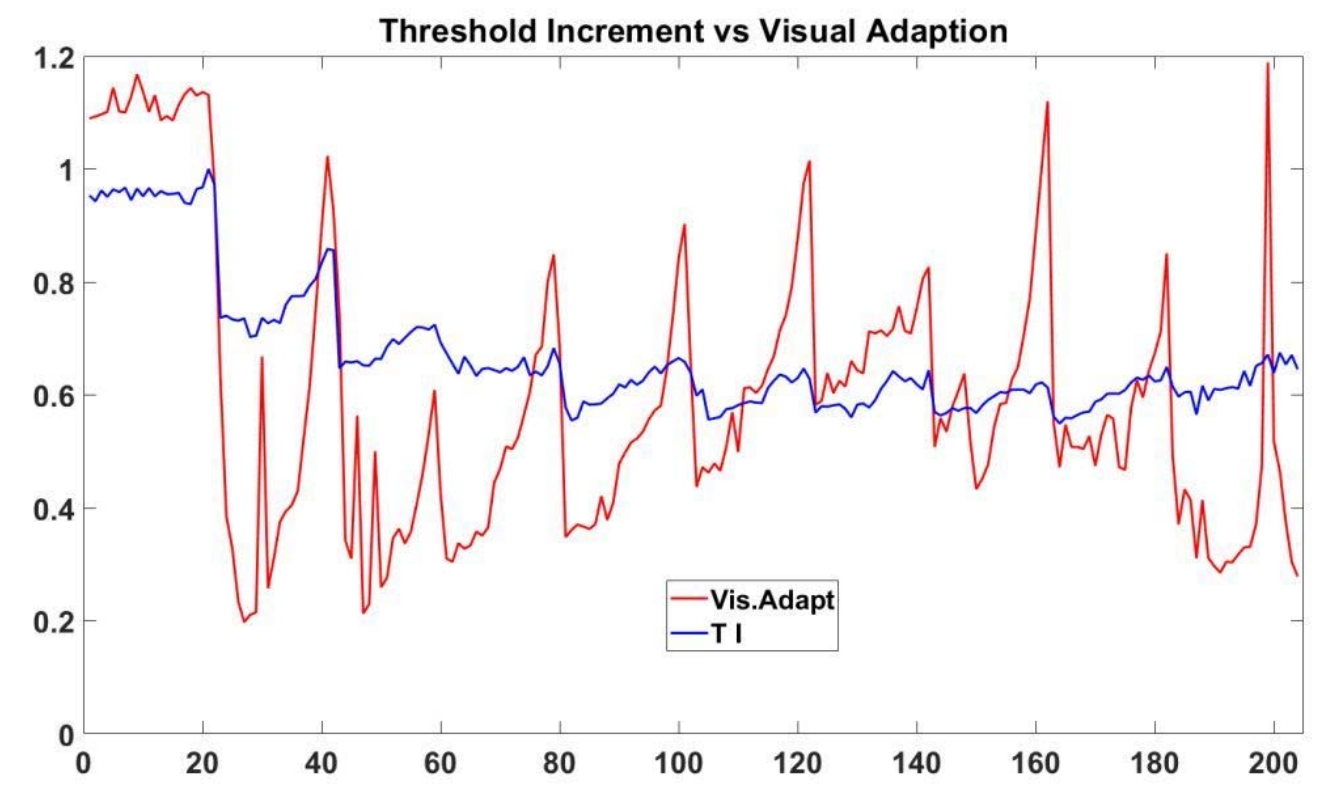

Figure 7 - Threshold Increment vs Visual Adaption along the boulevard

\section{Conclusion and outlook}

A High Dynamic Range - Imaging Luminance Measuring Device is developed based on four CMOS cameras. Geometric and photometric calibrations are described as well as HDR reconstruction process. To evaluate glare along a route, two models are performed on luminance maps. Threshold Increment model takes into account the sources excentricity, so needs a gaze point and is defined as a static model. The question of its validity in moving situation should be determined. A second model based on visual adaption is presented. Natively it is a dynamic model, but does not consider sources excentricity. The question of its relevancy as a quantitative glare model is also asked. Future experimentations are planned to answer these questions. While a target detection task, drivers will subject different glare situations. Recorded data should confirm or invalidate the use of both models for evaluating glare along a route. 


\section{References}

BOUCHER, V. GREFFIER, F. and FOURNELA, F. 2015. Disability Glare Evaluation in Driving Condition using High Dynamic Range Images. Proceedings of 28th CIE Session. 209216.

BOUCHER, V. GREFFIER, F. FOURNELA, F. DRONNEAU, R. DUMONT, E. 2017. High Dynamic Range Imaging Luminance Measuring Device (HDR-ILMD) and applications in motion. Proceedings of CIE 2017 Midterm Meetings and Conference on Smarter Lighting for Better Life.

CIE 1976. CIE 31-1976. Glare and Uniformity in Road Lighting. Vienna: CIE.

CIE 2002. CIE 146-2002. CIE COLLECTION on GLARE. Vienna: CIE.

DECKER, S.J. McGRATH, R.D. BREHMER, K. and SODINI, C.G. 1998. A 256x256 CMOS imaging array with wide dynamic range pixels and column-parallel digital output. IEEE J. of Solid State Circuits, 33, 2081-2091.

GREFFIER, F. CHARBONNIER, P. TAREL, J.P. BOUCHER, V. and FOURNELA, F. 2015. An automatic system for measuring road and tunnel lighting performance. Proceedings of 28th CIE Session. 1647-1656.

KAVADIAS, S. DIERICKX, B. SCHEFFER, D. ALAERTS, A. UWAERTS, and D. BOGAERTS, J. 2000. A Logarithmic Response CMOS Image Sensor with On-Chip Calibration. IEEE J. of Solid State Circuits, 25, 1146-1152.

KLEINFELDER, S. LIM,S.H. LIU, X.Q. and EL GAMAL, A. 2001. A 10,000 Frames/s 0.18um CMOS Digital Pixel Sensor with Pixel-Level Memory. Proc. of the 2001 IEEE International Solid-State Circuits Conference, 88-89.

KNIGHT, F.T. 1983. Design of an Integrated Optical Sensor with On-Chip Preprocessing. PhD thesis, MIT.

NAYAR, S.K. MITSUNAGA, T. 2000. High Dynamic Range Imaging: Spatially Varying Pixel Exposures. IEEE Conference on Computer Vision and Pattern Recognition (CVPR), 1, 472-479.

PATTANAIK, S.N. TUMBLIN, J. YEE, H. and GREENBERG, D.P. 2000. Time-Dependent Visual Adaptation For Fast Realistic Image Display. Proceedings of SIGGRAPH, 47-54.

PORSCH, T. WALKLING, A. SCHMIDT, F. SCHIERZ, C. Measurement of the threshold increment (TI) in road lighting based on using ILMD, CIE 2014. "Lighting Quality and Energy Efficiency“ Kuala Lumpur/Malaysia.

REINHARD, E., STARK, M., SHIRLEY, P. and FERWEDA J. 2002. Photographic tone reproduction for digital images. ACM Transactions on Graphics (Proc.SIGGRAPH), 21(3), 267-276.

REINHARD, E., WARD, G., DEBEVEC, P., PATTANAIK, S., HEIDRICH, W. and MYSZKOWSKI, K. 2010. High Dynamic Range Imaging. 2nd ed. Burlington: Morgan Kaufmann Publishers.

SAYAG, M. 1991. Non-linear Photosite Response in CCD Imagers. US Patent N5,055,667. 